\title{
FACTORES DE RIESGO EN INFECCIÓN Y COLONIZACIÓN POR Escherichia coli y Klebsiella pneumoniae PRODUCTORAS DE BETALACTAMASAS DE ESPECTRO EXTENDIDO
}

\author{
Adriana Jiménez MD*, Germán A. Carrero Forero MD**
}

\section{Resumen}

Las betalactamasas de espectro extendido (BLEE) son enzimas producidas por bacterias Gram negativas, que tienen la capacidad de destruir el anillo betalactámico de las cefalosporinas de tercera generación, permitiendo a la bacteria continuar con el entrecruzamiento del peptidoglicano y con la formación de pared celular sin alteraciones. Descritas en los años ochenta su diseminación y aumento en la incidencia en todos los continentes ha llevado al incremento de la morbilidad y mortalidad de los pacientes, prolongación de los días de estancia hospitalaria, mayor demanda de uso de carbapenémicos e incremento de los costos de la atención en salud. Predecir el patrón de resistencia del microorganismo que infecta a un paciente con base en el análisis de los factores de riesgo asociados permite la óptima elección de la antibioticoterapia empírica, racionalizando el uso de los antibióticos de amplio espectro disponibles y mejorando la sobrevida de los pacientes. Para esta revisión se realizó una búsqueda de los estudios de casos y controles en los cuales se investigaron los factores de riesgo asociados con infección o colonización por bacterias productoras de BLEE a saber: exposición previa a antibióticos, estancia en UCI, uso de catéter vesical, catéter central o ventilación mecánica.

Palabras clave: factores de riesgo, antibiótico, resistencia, betalactamasas de espectro extendido. Abreviaturas: BLEE, betalactamasas de espectro extendido; UCI, unidad (es) de cuidados intensivos.

\section{RISK FACTORS IN EXTENDED-SPECTRUM BETALACTAMASE PRODUCING ESCHERICHIA COLI AND KLEBSIELLA PNEUMONIAE INFECTION AND COLONIZATIÓN}

\begin{abstract}
Extended-spectrum betalactamases (ESBLs) are enzymes produced by gram- negative bacteria which are capable of destroying the betalactamic ring of third-generation cephalosporins enabling the bacteria to continue the peptidoglican crosslinking process resulting in unaltered integrity of the bacterial cell wall. ESBLs were first detected in the 1980s. Its incidence increased and dissemination in all continents has lead to greater morbidity and mortality rates, prolonged hospitalization, greater usage of carbapenems and rising health care costs. Prediction of resistance patterns of infecting microorganisms based on the analysis of the risk factors associated facilitate optimal selection of empirical antibiotic treatment, rationalized use of available broad spectrum antibiotics and improvement of patient survival. This review searched for case studies and follow up trials which investigated the risk factors associated with ESBL producing bacterial infection or colonization, that is, previous exposure to antibiotics, hospitalization at the ICU, urinary catheterization, central venous catheterization and mechanical ventilation.
\end{abstract}

Key words: risk factors, antibiotic, resistance, extended-spectrum betalactamases.

Fecha recibido: enero 20 de 2012 - Fecha aceptado: julio 7 de 2012

* Profesora Asociada, Fundación Universitaria de Ciencias de la Salud. Jefe del Comité de Infecciones, Hospital de San josé. Bogotá DC. Colombia.
** Residente III de Medicina Interna, Fundación Universitaria de Ciencias de la Salud, Bogotá DC, Colombia. 


\section{Introducción}

Las betalactamasas son enzimas producidas por múltiples microorganismos capaces de alterar la estructura y función de los betalactámicos. Desde 1940 se encuentran artículos evidenciando un número diverso y creciente de moléculas que han sido informadas desde entonces. En 1983 se inician los primeros reportes de betalactamasas de espectro extendido (BLEE) ${ }^{1}$ capaces de degradar varios betalactámicos de diferente espectro, dificultando el éxito del tratamiento antibiótico y aumentando la mortalidad y el costo médico. ${ }^{2}$ Estas BLEE son producidas principalmente por enterobacterias, las más relevantes son E.coli y K. pneumoniae. ${ }^{3}$ Hasta el momento se ha reportado un número superior a 200 moléculas y se conocen varios intentos para clasificarlas. ${ }^{4}$ Las BLEE pertenecen al grupo 2 be de la clasificación funcional de Bush y Jacoby; 2b por el sustrato que son las penicilinas y las cefalosporinas, inhibidas no por EDTA (metales quelantes), sino por inhibidores de betalactamasas y tienen hidrolósis similar a las bencilpenicilinas y cefalosporinas. La $\underline{\mathbf{e}}$ por ser de espectro extendido, incrementando la capacidad de hidrólisis a las oximino- $\beta$ lactámicos (cefotaxima, ceftriaxona, cefepime, aztreonam). Las enzimas más representativas son las derivadas de TEM, SHV y CTX-M; menos frecuentes las BES, PER, VEB y TLA.

Estrategia de búsqueda: se realizó la búsqueda de los artículos de PUBMED utilizando términos MeSH: risk factors, antibiotic, resistance, extended spectrumbeta lactamase. Se seleccionaron para esta revisión los estudios retrospectivos de casos y controles o de cohorte en idioma inglés o español y se revisaron un total de 19 artículos.

Europa reporta gran variación de la prevalencia en UCI de cepas productores de BLEE: $3 \%$ en Suiza, $34 \%$ en Portugal y $58 \%$ en Turquía. ${ }^{5,6} \mathrm{En}$ los años 90 Francia llegó a una prevalencia de cerca de $19 \%$, pero después de los programas de control de infección instaurados en sus instituciones, la cifra para el 2000 bajó a $7.9 \% .^{7}$

Los primeros reportes en Norteamérica vienen de 1988. ${ }^{8}$ En 2002 el 75\% de las instituciones de Esta- dos Unidos ya tenían cepas productores de BLEE', esta cifra varía de acuerdo con el sitio evaluado en la institución, hallando hasta $25 \%$ en las UCI, $5.7 \%$ en áreas fuera de ellas y $1.8 \%$ en la comunidad. ${ }^{10}$

En África no se cuenta con estudios de vigilancia nacionales, sin embargo en algunas instituciones hay prevalencias tan altas como 36.1\%. ${ }^{11}$ Australia reporta una cercana al 5\%. ${ }^{11}$ Estudios de vigilancia nacional en Asia revelan amplias diferencias según los países: 5 a 8\% en Corea, Japón y Malasia, 12 a 24\% en Tailandia, Singapur y Taiwán. ${ }^{12,13}$

América Latina tiene la prevalencia más alta del mundo de $K$. pneumoniae productora de BLEE (45 a $51.9 \%$ ), para E. coli 8.5 a $18.1 \% .{ }^{14}$ Los primeros informes fueron en 1987 en Chile. ${ }^{1}$ Colombia, Brasil y Venezuela tienen tasas entre 30 y $60 \%$ de BLEE ${ }^{12,15-20}$ mientras en Chile y Uruguay son 4.5 y $12 \% .^{1}$ Estas diferencias están relacionadas con la condición económica del país, el número de pacientes por enfermera en los hospitales, el uso no controlado de antibióticos y la higiene hospitalaria. ${ }^{1}$

En Colombia se inician los primeros reportes de BLEE en 1999 con valores por encima del 40\%, siendo mayor en la costa atlántica $37.6 \%$ con respecto a la zona andina, $10 \%$ y $30 \%$ para E. coli y K. pneumoniae..$^{21,22}$

Las infecciones por enterobacterias productoras de BLEE, así como el inicio de terapia empírica inapropiada han cursado con una mayor tasa de mortalidad. Así Tumbarello la documentó cuatro veces mayor en quienes recibieron una terapia antibiótica inicial inapropiada. ${ }^{23}$ La mortalidad en un estudio brasileño fue para los pacientes infectados por cepas productoras de BLEE $51 \%$ frente al $29 \%$ de las enterobacterias no productoras. ${ }^{24}$ Cordery y col. anotan un riesgo 3.9 veces mayor de mortalidad en infectados por enterobacterias productoras de BLEE con respecto a los controles. ${ }^{25}$

Dada la importancia del tema, la creciente aparición de nuevas cepas multirresistentes y el escaso arsenal terapéutico con el que se cuenta, consideramos relevante determinar y realizar una búsqueda activa de los factores que predisponen al paciente para adquirir este 
tipo de microorganismo productor de BLEE.

\section{Asociación entre dispositivos médicos y aislamientos de bacterias BLEE}

\section{Catéter urinario}

La sonda vesical es un dispositivo invasivo de amplio uso en nuestras instituciones de salud para la monitoría de pacientes en estado crítico o con uropatía obstructiva baja. Sin embargo, en muchas ocasiones la indicación y la duración no están tan claras para el médico tratante. Su empleo explica en parte el desarrollo de infecciones urinarias asociadas con el cuidado médico: $40 \%$ de las infecciones hospitalarias y $23 \%$ de las de UCI. ${ }^{26}$ Todos los mecanismos de defensa para disminuir el riesgo de infecciones urinarias se alteran por la presencia del catéter vesical: cambia el $\mathrm{pH}$ urinario, disminuye el contacto de la bacteria con el epitelio urinario y por tanto los efectos de las proteínas de Tamm-Horsfall ${ }^{27}$ facilita la formación de biopelículas intra y extraluminales en el catéter, lo que disminuye los efectos de las células inflamatorias y la terapia antibiótica sobre los microorganismos, permitiendo la migración ascendente de estos. La bacteriuria asociada presenta una incidencia aproximada de 3 a $8 \%$ por día de catéter, es decir que al mes el $100 \%$ de los pacientes con el presentarán bacteriuria. ${ }^{28-32}$ Otros factores que facilitan la infección del catéter son la deficiencia en el cuidado y manipulación del mismo, la técnica para colocarlo, el sitio en el que se realiza el procedimiento, la edad, padecer diabetes mellitus y presentar valores elevados de creatinina. ${ }^{28,28,30,33-36}$

\section{Catéter venoso central}

Es un dispositivo de uso frecuente en el paciente crítico o con neoplasias hematológicas. Es responsable de una gran proporción de infecciones en el torrente sanguíneo y se ha asociado con aumento de mortalidad, costos y estancia prolongadà. ${ }^{37-39}$ Es más prevalente la bacteremia asociada con catéter en UCI que en salas generales. ${ }^{40}$ La colonización es el primer paso para que después ocurra la infección y esta se da de muchas maneras, en especial la migración de microorganismos por ruta extraluminal (interfase piel-catéter) y por contaminación del lumen. La piel es la fuente de los microorganismos que contaminan en forma temprana el catéter (<10 días), mientras los que se contaminan de manera tardía tienen su origen en las líneas venosas que se usan para acceder al catéter. ${ }^{41}$ Con menor frecuencia ocurre colonización por bacteremia secundaria a otro foco infeccioso. ${ }^{42}$ Los factores de riesgo de infección del torrente sanguíneo asociada con catéter central son la hospitalización larga, la cateterización prolongada, el uso de la vía yugular interna, la neutropenia y la nutrición parenteral.

\section{Ventilación mecánica}

Es una herramienta útil en el manejo de los pacientes en estado crítico, pero conlleva mayor riesgo de morir por procesos infecciosos de origen hospitalario. El $86 \%$ de las neumonías nosocomiales se asocian con ventilación mecánica, cuya mortalidad puede alcanzar 50\% ${ }^{43-46}$, aumenta la estancia hospitalaria entre cuatro y trece días e incrementa los costos invertidos en su diagnóstico y tratamiento. ${ }^{47-49} \mathrm{La}$ relación entre el tiempo de ventilación mecánica y la probabilidad de desarrollar neumonía está bien establecida, encontrando incidencias que progresan $3 \%$ por día para los primeros cinco días, $2 \%$ por día los siguientes cinco y $1 \%$ por día los siguientes cinco días. ${ }^{50} \mathrm{El}$ tracto respiratorio está constituido en forma básica por las fosas nasales, sus vibrisas y mucosa, la naso y orofaringe, la tráquea con su epitelio ciliado y su capa de moco, todas trabajan en conjunto para reducir el acceso de microorganismos y partículas a nivel alveolar. El paciente en estado crítico bajo ventilación mecánica, sedación e intubación orotraqueal pierde todas estas defensas y las partículas y microorganismos acceden más fácil a los pulmones, no puede toser, se elimina el barrido ciliar del epitelio, la boca permanece seca y facilita el crecimiento de placa bacteriana. La causa principal de neumonía asociada con ventilación mecánica tiene relación directa con microaspiración de bacterias ${ }^{51-54}$ y sumado a esto el tamaño del inóculo nos lleva al desarrollo de colonización del tracto 
respiratorio y su posterior proceso infeccioso con los desenlaces antes mencionados. ${ }^{55}$

La relación entre estos dispositivos y las enterobacterias productoras de BLEE se pudo establecer en múltiples estudios de casos y controles realizados en diferentes países (Tabla 1). Mendelson y col. publican un estudio realizado en un centro geriátrico y encuentran una asociación con significación estadística al usar sonda vesical permanente (OR 2.74 IC 95\% 1.43-5.3). ${ }^{56}$ En Taiwán, un hospital universitario de 2.500 camas realizó un estudio de bacteremia por $E$. coli productora de BLEE encontrando los resultados siguientes en el análisis univariado: catéter urinario (OR 7.68 IC 95\% 3.02-19.3), catéter venoso central (OR 5.38 IC 95\% 2.67-10.86) y ventilación mecánica (OR 17 IC 95\% 2.29-137.59), gastrostomía y la sonda nasogástrica (OR 3.79 IC95\% 1.72-8.36).
Sin embargo, en el análisis multivariado, solo el uso de catéter urinario alcanzó la significación estadística. ${ }^{57}$ Un estudio multicéntrico realizado en España por Rodríguez-Baño y col. en el que participaron trece instituciones, investigaron bacteremia por $E$. coli de origen en la comunidad, hallando diferencia con estadística significativa entre casos y controles para el factor de riesgo "uso de catéter urinario" (OR 3.1 IC 95\% 1.5-6.5), pero no encontró relación con el uso de catéteres venosos (OR 1.6 IC 95\% 0.6-4.2) y no se exploró la asociación con la ventilación mecánica. ${ }^{58}$ Este autor en el mismo año en un estudio similar sobre factores de riesgo asociados con bacteremia de origen nosocomial, no encontró ninguna asociación con el uso de dispositivos médicos. ${ }^{59}$ Rodriguez Baño en un estudio previo realizado en un solo hospital y publicado en 2008, encontró asociación entre el uso de catéter urinario y la bacteremia por E.coli: BLEE positivo. ${ }^{60}$

\begin{tabular}{|c|c|c|c|c|c|c|}
\hline \multirow{2}{*}{ Estudio } & \multicolumn{2}{|c|}{ Sonda vesical } & \multicolumn{2}{|c|}{ Catéter central* } & \multicolumn{2}{|c|}{ Ventilación mecánica } \\
\hline & OR & IC & OR & IC & OR & IC \\
\hline Chaiwarith (6I) & NR & & NR & & 1.95 & $1.17-3.25$ \\
\hline Cheol In Kang (64) & NS & & NR & & NR & \\
\hline Cheol In Kang (65) & NS & & NS & & NS & \\
\hline Cordery (25) & NS & & NS & & NS & \\
\hline E. Hyle (66) & NS & & NS & & NS & \\
\hline Fernández Quirante(67) & NS & & NR & & NS & \\
\hline G. Mendelson (56) & 2.7 & $1.4-5.3$ & NE & & NE & \\
\hline Kuster (63) & NS & & NS & & 10.56 & $1.06-579.1$ \\
\hline MF Lin (62) & NS & & NS & & NS & \\
\hline Pak Leung Ho (68) & NS & & NR & & NR & \\
\hline Peña (69) & NS & & NS & & NS & \\
\hline Rodriguez-Baño (60) & 3.9 & $1.1-13.7$ & NS & & & \\
\hline Rodriguez-Baño (59) & NS & & NS & & NS & \\
\hline Rodriguez-Baño 2010 (58) & 3.1 & $1.5-6.5$ & NS & & NE & \\
\hline Tumbarello (23) & NS & & NR & & NS & \\
\hline Un-In Wu (57) & 6,21 & $1.91-20.5$ & NS & & NS & \\
\hline Vargas Superti (24) & NS & & NS & & NS & \\
\hline
\end{tabular}

*NR, no reportado; NS, no significativo; NE,no evaluado. 
El hospital Chiang Mai en Tailandia publicó los resultados de un estudio realizado entre 2005 y 2006, donde encuentran como único dispositivo relacionado con BLEE la ventilación mecánica (OR 1.95 IC 95\% 1.17-3.25) ${ }^{61}$ Lin y col. realizaron en 2001 un estudio encontrando diferencias estadísticas en la exposición a sonda vesical (OR $4.88 \mathrm{p}<0.001)$, catéter central (OR3.9 p<0.001), sonda nasogástrica (OR 6.5 p >0.001) y traqueostomía (OR $6.44 \mathrm{p}<0.001$ ). Sin embargo, al realizar el análisis multivariado solo encontraron como factor de riesgo independiente la traqueostomía (OR 5.13 IC 95\% 1.24-21.1). ${ }^{62} \mathrm{El}$ estudio realizado por Kuster en Suiza, un país con baja prevalencia de aislamientos de bacterias productoras de BLEE, encontró asociación entre la ventilación mecánica y la infección por bacterias productoras de BLEE (bacteremia, infección de vía urinaria y relacionada con catéter venoso central). No se encontró asociación con el uso de otros dispositivos. ${ }^{63}$

Otros estudios han explorado la asociación entre dispositivos médicos y la infección por bacterias BLEE sin encontrar diferencias con significancia estadística entre los casos y controles. De los estudios con resultados negativos se encuentran el de Cordery realizado en el Reino Unido en pacientes con bacteremia hospitalizados en $\mathrm{UCI}^{25}$, los de Cheol In Kang en Corea y publicados en el 2010 y 2011 sobre factores de riesgo para bacteremia de origen en la comunidad y en pacientes con neoplasia hematológicas ${ }^{64.65}$, el de Hyle en Estados Unidos diseñado para identificar factores de riesgo asociados con la infección por bacterias BLEE multirresistentes con resistencia adicional a trimetoprin-sulfamethoxazole, aminoslucósidos y quinolonas ${ }^{66}$, el estudio de Quirante realizado en España en pacientes con bacteremia ${ }^{67}$, y el publicado en 2002 por Park-Leung Ho realizado en China en pacientes con bacteremia por E. coli $\mathrm{BLEE}^{68}$, el de Peña publicado en 2006 y realizado en un hospital en España en pacientes con aislamientos de $E$. coli BLEE en cualquier muestra ${ }^{69}$, el conducido por Tumbarello entre 1999 y 2003 en un hospital de Roma en pacientes con bacteremia por Klebsiella pneumoniae y finalmente el de Vargas Superti realizado entre 2000 y 2006 en un hospital de Porto Alegre (Brasil) también en pacientes con bacteremia por $E$. coli o Klebsiella $s p .^{23,24}$

\section{Asociaciónentreantibioticoterapia y aislamientos de BLEE}

El uso de antibióticos ha modificado de forma radical la práctica de la medicina, dada la mayor sobrevida de los pacientes con infecciones de origen bacteriano. Sin embargo son un arma de doble filo, nos muestra por un lado los beneficios en morbilidad y mortalidad pero además en el corto plazo vemos la toxicidad hepática y renal que su uso implica y en el largo plazo la aparición acelerada de microorganismos resistentes a esta terapia. Los médicos, aún hoy día, no se detienen a considerar la importancia de conservar antibióticos de amplio espectro para su uso en el escenario correcto. La aparición de cepas resistentes es un ejemplo claro de la selección natural descrita por Darwin y su aumento con el paso del tiempo es algo inevitable. Varias formas de resistencia están descritas en la literatura, pero su revisión se escapa del enfoque de este artículo. Sabemos que el uso, sobreuso y abuso del arsenal antibiótico es el mecanismo de presión selectiva más deletéreo en la aparición de cepas multirresistentes. Cuantificar la relación entre el volumen de antibiótico consumido y la frecuencia de aparición de resistencias será una tarea difícil.

La asociación entre el uso de terapia antibiótica previa y el aislamiento de microorganismos productores de BLEE ha sido documentado en múltiples estudios que están resumidos en la Tabla 2.

Se encontró relación estadística significativa entre el uso previo de antibióticos (sin discriminar por tipo) y la infección por E-coli o Klebsiella sp. productoras de BLEE en el análisis multivariado de los estudios de factores de riesgo de Quirante (OR $=2.29)^{67}$ García (OR $=2,61)^{70}$, Mendelson $(\mathrm{OR}=2,94)^{56}$, Peña $(\mathrm{OR}=3,9)^{70}$, Tumbarello $(\mathrm{OR}=11,8)^{23}$, Un In Wu $(\mathrm{OR}=2,93)^{57} \mathrm{y}$ en el de Rodríguez Baño de bacteremia de origen en la comunidad $(\mathrm{OR}=2,7) .{ }^{58}$ De los anteriores estudios, solo el de Un-In Wu encontró asociación con el uso previo de un tipo específico de antibiótico, específicamente cefalosporinas de tercera generación $(\mathrm{OR}=5,16)$.

Otro estudios han investigado sobre la asociación con un tipo particular de antibióticos sin haber encontrado 
Tabla 2. Análisis multivariado de los estudios sobre uso de antibiótico previo y la adquisición de cepas productoras de BLEE

\begin{tabular}{|c|c|c|c|c|c|c|c|c|}
\hline \multirow{2}{*}{ Estudio } & \multicolumn{2}{|c|}{ Cefalosporinas } & \multicolumn{2}{|c|}{ Quinolonas } & \multicolumn{2}{|c|}{$\beta$-lactámico más inhibidor } & \multicolumn{2}{|c|}{ Uso de antibiótico previo } \\
\hline & OR & IC & OR & IC & OR & IC & OR & IC \\
\hline Bin Du (72) & 4.1 & $1.4-11.8$ & NS & & NS & & NR & \\
\hline Chaiwarith (6I) & 3,92 & $2.72-6.77$ & NR & & NR & & NR & \\
\hline Cheol-In Kan (64) & NS & & NS & & NR & & NS & \\
\hline Cheol-In Kan (65) & NS & & NS & & NR & & NR & \\
\hline Cordery (25) & NE & & NE & & NE & & NS & \\
\hline E. Hyle (66) & NS & & NS & & NS & & NS & \\
\hline Fernández Quirante (67) & NS & & NS & & NR & & 2.29 & $1.35-3.88$ \\
\hline Kuster (63) & NR & & NR & & NR & & 2.88 & $1.13-8.49$ \\
\hline MF Lin (62) & 13.4 & $1.21-148.5$ & NS & & NR & & NS & \\
\hline Mendelson (56) & NS & & $\mathrm{S}$ & & NS & & 2.94 & $1.4-5.39$ \\
\hline Pak Leung Ho (68) & NS & & NR & & NS & & NR & \\
\hline Peña (69) & NS & & NS & & NS & & 3,9 & $2.0-7.8$ \\
\hline Rodriguez- Baño (60) & 3.9 & $1.1-14.1$ & 6.2 & I.8-20.7 & NR & & NS & \\
\hline Rodriguez- Baño (59) & 6 & $3-11.8$ & NS & & NR & & NS & \\
\hline Rodriguez- Baño (58) & NS & & NS & & NE & & 2.7 & $1.5-4.9$ \\
\hline Tumbarello (23) & NR & & NR & & NR & & 11.8 & 2.72 to 51.08 \\
\hline Un-In-Wu (57) & 5.16 & $1.03-25.79$ & NS & & NS & & 2.93 & $1.18-7.30$ \\
\hline Vargas Superti (24) & NS & & NS & & $6.2^{*}$ & I.I-34.7 & NS & \\
\hline Wener (73) & 1.89 & $0.9-3.78$ & 2.8 & $1.4-5.9$ & 10.1 & $1.2-86.92$ & NR & \\
\hline
\end{tabular}

* Piperacilina/tazobactam; NR; no reportado; NS, no significativo; NE, no evaluado.

o no explorando la asociación en general. Supertir y colaboradores en Basil, no encontraron diferencias significativas entre casos y controles con el uso previo de cefalosporinas de tercera generación (oximinocefalosporinas), cefepime, quinolonas y carbapenémicos, sin embargo la diferencia si fue significativa con el uso previo de piperacilina tazobactam $(\mathrm{OR}=6,2){ }^{24}$ El estudio de Harris de la Universidad de Maryland en pacientes hospitalizados en UCI encontró asociación entre el uso previo de vancomicina $(\mathrm{OR}=2,11$ IC $95 \% 1,34-3.31)$ y piperacilina tozobactam $(\mathrm{OR}=$ 2.05 IC 85\% 1-3- 3.1) y la colonización por E.coli o Klebsiella sp. al momento de la admisión. ${ }^{71} \mathrm{M}-\mathrm{F}$ Lin y col. en el Hospital Hsin-Chu de Taiwan documentaron como único factor de riesgo el uso de ceftazidime (OR 13,4 ) aunque el intervalo de confianza es muy amplio CI.21-148.5). ${ }^{62}$
La revista médica de cuidado intensivo en 2002 publica un estudio de casos y controles en pacientes con bacteremia, encontrando como único factor de oximinocefalosporinas (OR 4.4 IC 95\% 1.4 - 11.8). ${ }^{72}$ Chaiwatith encontró significativa la asociación con el uso previo de cefalosporinas (3.92) ${ }^{61}$ Los estudios de Rodríguez Baño publicados en 2010 (asociación con neumonía nosocomial) y 2008 encontraron asociación con el uso previo de oximinocefalosporinas (OR 6,0 y 3,9 respectivamente) y con el uso de quinolonas (OR 1,8); este último hallazgo solo el estudio del 2008. ${ }^{59,60}$ Por último el estudio de Wener realizado en Boston (Estados Unidos) entre 1933 y 2001 encontró como factor de riesgo asociado con el aislamiento de Klebsiella sp. productora de BLEE el uso previo de $\beta$ lactámico más inhibidor (OR 10,17) y quinolonas (OR 2.85) la asociación con oximinocefalosporinas solo fue significativa en el subgrupo de pacientes no tratados 
con quinolonas. ${ }^{73} \mathrm{El}$ resto de estudios revisados solo encontró asociaciones en el análisis univariado.

Cheol Inkan en Sur Corea con el estudio nacional de vigilancia para bacteremia, buscó factores de riesgo en bacteremia por $E$. coli productora de BLEE que provinieron de la comunidad. Aunque se encontraron diferencias estadísticas significativas con el uso especifico por grupo de antibiótico en el análisis univariado (cefalosporinas, penicilinas, fluoroquinolonas reportaron $\mathrm{p}<0.001$ ), no hubo resultados significativos en el análisis multivariado con regresión logística. ${ }^{64}$ El estudio de Emily Hyle y col. no reportó ninguna relación clínica significativa con el uso de antibióticos previos al aislamiento de cepas productoras de BLEE: trimetoprin-sulfametoxasol (OR 0.63 IC 95\% 0.3-1.33), aminoglucósidos (OR 1.42 IC 95\% 0.8-2.5), cefalosporinas de tercera generación (OR 1.28 IC 0.483.5), betalactámico más inhibidor (OR 1.18 IC 95\% 0.6-2.3) y con fluoroquinolonas (OR 1.85 IC 95\% 1.1-3.2) ${ }^{66}$ Estas últimas no alcanzaron la significancia estadística en el multivariado. El estudio español sobre bacteremia de origen en la comunidad encontró en el análisis univariado a las cefalosporinas (OR 13.4 IC 95\% 2.9-61.4) y las quinolonas (OR5.6 IC 95\% 2.512.5) como factores de riesgo para cepas productores de BLEE, sin embargo al realizar el análisis multivariado las quinolonas (OR 2.8 IC 95\% 1.2-6.5) fueron el único grupo terapéutico identificado como factor de riesgo. ${ }^{66}$ En el estudio del Hospital universitario de Taiwán en 2010, se encontró mayor exposición a quinolonas $(\mathrm{p}<0.001)$ y oximinocefalosporinas $(\mathrm{p}<0.001)$ en casos que en los controles, con cepas de E. coli en sangre productoras de BLEE; en el análisis multivariado no se halló relación estadística y clínica significativa en relación con las oximinocefalosporinas (OR 0.22 IC 0.1-0.6). ${ }^{57}$

\section{Asociación entre comorbilidades y aislamientos de BLEE}

Con el progreso en la invèstigación de las enfermedades muchas de ellas terminan siendo un proceso de inflamación injuria crónica, que llevan al paciente al estrés fisiológico el cual al final altera la función de los linfocitos y las células asesinas naturales ( $n a$ tural killer). Este estado inflamatorio crónico está relacionado con el mayor riesgo de infecciones. Toda comorbilidad crónica en el paciente lo predispone a adquirir infecciones (bacterianas, micóticas o virales), lo lleva a mayor contacto con el medio hospitalario y lo predispone al uso de antibiótico. Lo mismo sucede cuando alteramos con fármacos el buen funcionamiento inmunológico, lo que también facilita los procesos infecciosos.

Las comorbilidades como factor de riesgo para adquirir una infección por bacterias productoras de BLEE han sido investigadas en varios estudios, sin embargo en muy pocos se ha encontrado la significancia estadística. En bacteremia por $E$. coli productora de BLEE, Cheol In Kang y colaboradores encontraron la enfermedad hepática (OR 2.0 IC 95\% 1-12 3-8) como única comorbilidad relacionada. ${ }^{64} \mathrm{En}$ infecciones de vía urinaria por E. coli y Klebsiella $s p$. productora de BLEE, el estudio de Mendelson en Israel encontró la anemia como factor de riesgo independiente (OR 3-2 IC $95 \% 1.6-6.5) .{ }^{56}$

Otros estudios han encontrado asociaciones solo en el análisis univariado, a continuación se presentan algunos de los factores de riesgo asociados. Velasco y col. no encontraron diferencias estadísticas en diabetes mellitus, insuficiencia renal, cirrosis, neoplasia sólida o inmunosupresión en relación con infección urinaria bacterémica por $E$. coli productor de BLEE. ${ }^{74} \mathrm{En}$ bacteremia por $E$. coli productora de BLEE de origen en la comunidad se encontró la enfermedad pulmonar crónica (OR 3.1 IC 95\% 24-67) como única entidad nosológica relacionada con cepas productoras de BLEE. ${ }^{58}$ Un-In Wu en Taiwán no encontró ninguna enfermedad crónica asociada con infección por E.coli productora de BLEE en sangre, pero la inmunosupresión si tuvo diferencias clínicas y estadísticas (OR 2.0 IC 95\% 1.12-3.9). ${ }^{57}$ En relación con infecciones por $K$. pneumoniae productora de BLEE, el estudio de Wener y col. reportó que las enfermedades respiratorias (OR 5.9 IC 95\%3.3-10.4), las afecciones hepáticas (OR 2.3 IC 95\% 1.1-5.1), la insuficiencia renal (OR 4.6 IC 95\% 2.2-9.5) y el cáncer (OR 1.6 IC $95 \%$ 1.1-2.4) predisponían a infecciones por este mi- 
croorganismo. ${ }^{73}$ Lin y col. en Taiwán, así como García y col. en España no encontraron ninguna diferencia estadística en comorbilidades en pacientes con infección por K.pneumoniae productora o no de BLEE. ${ }^{62,70}$ Ninguna comorbilidad fue asociada en bacteremia por enterobacterias productoras de BLEE en pacientes con malignidad hematológica. ${ }^{65}$

\section{Asociaciones misceláneas}

Dentro de los estudios analizados se reportan algunas asociaciones que no fueron frecuentes, pero consideramos importante describirlas en esta revisión. Las infecciones asociadas con el cuidado médico (OR 1.86 IC 95\% 1.44-2.39) se relacionaron con el aislamiento de $E$. coli en sangre de origen en la comunidad ${ }^{64}$ Esta misma variable fue encontrada en el estudio de Cheol Ioskary en bacteremia en paciente con neoplasia hematológica (OR- 5.66 ic 95\% $1.6-20.3)^{65}$, en el de Rodríguez Baño en pacientes con bacteremia de origen en la comunidad (OR 2.9 IC $95 \% 1.23-8)^{58}$ y en el de Pak-Leung realizado en China en bacteremia con E.coli, $\left(\mathrm{OR}=16.5\right.$ IC 95\% 5-6 - 49). ${ }^{68}$ La relación con el género femenino solo se encontró en el estudio de Peña. ${ }^{69}$ La estancia en hogar geriátrico (OR 6.5 IC 95\% 1.4-30.9) se asoció con infecciones urinarias bacterémicas por $E$. coli productora de BLEE. ${ }^{74}$ Wener reportó la edad $>65$ años (OR 1.73 IC 95\%1.3-2.31) como factor de riesgo para este tipo de aislamientos en Klebsiella sp. ${ }^{73}$ Esta misma variable (edad $>60$ años) fue reportada como riesgo de colonización por bacterias productoras de BLEE (OR 1.79 IC95\% 1.24-2.6) en el estudio de Harris en UCI. ${ }^{71}$

\section{Conclusiones}

Las betalactamasas de espectro extendido (BLEE) son enzimas que hidrolizan las oxy-imino cefalosporinas (cefalosporinas de $3^{\text {a }}$ generación) y que son neutralizadas in vitro por los inhibidores de $\beta$-lactamasas como el sulbactam o el ácido clavulónico y el cefoxitin, una cefalosporina de segunda generación. Inicialmente identificadas en Alemania, en los inicios de los 80s, su rápida diseminación favorecida por la transmisión por medio de plásmidos ha llevado a que en la actualidad se hayan identificado alrededor de 200 variantes en diferentes puntos geográficos del planeta. El aumento en la incidencia de infecciones por Klebsiella pneumoniae y Escherichia coli productoras de BLEE constituye una amenaza para el óptimo tratamiento de los pacientes, debido a que en varios casos no se inicia el tratamiento empírico adecuado o se elige el antibiótico inapropiado seleccionado con base en una interpretación errada de los resultados del antibiograma.

Lograr evaluar de manera adecuada los pacientes que debutan con una infección y predecir la probabilidad de que ésta sea causada por una bacteria productora de BLEE requiere de la comprensión de los factores de riesgo asociados. Los primeros estudios sobre factores de riesgo asociados al aislamiento de bacterias productores de BLEE aparecen publicados a finales de los 90s y el número de publicaciones aumenta en proporción al aumento en la prevalencia del fenómeno, de tal forma que entre 2011 y 2012 en la base de datos de pubMed se encuentran nueve artículos sobre el tema provenientes de todos los continentes.

En la presente revisión se analizaron 19 estudios retrospectivos de casos y controles que buscaron asociaciones entre diferentes variables de exposición y la infección o colonización de cualquier origen por bacterias BLEE positivas. Fueron realizados en España, Suiza, Italia, Inglaterra, Estados Unidos, Tailandia, Corea, Taiwan, China, Israel e India. No se encontró ningún estudio proveniente de Latinoamérica.

El factor de riesgo para el cual se encontró más asociaciones (42\% de los estudios) con significancia estadística en el análisis multivariado fue el uso de antibiótico previo (rango de OR de 2.2 a 11,8); en segundo lugar ( $31 \%$ de los estudios) el antecedente de tratamiento con cefalosporinas de tercera generación (Rango de OR de 3.7 a 13.4) y en tercer lugar (asociación encontrada en $26 \%$ de los estudios) el empleo de catéter vesical (rango de OR entre 2.7 y 6.21).

La asociación con mayor magnitud ( $\mathrm{OR}=27,01$ IC 95\% 2.38-1733.28) se encontró para la variable "inicio de síntomas o antibiótico previo en país extranjero" 
en el estudio de Kuster realizado en Suiza, un país con baja prevalencia de BLEE. Esta variable solo fue analizada en este estudio. De acuerdo con la magnitud de la asociación, el estudio de Kuster es seguido por el de Pak Leung Ho realizado en China entre 1996 y 1998 que encontró en el multivariado una significancia estadística para la variable origen nosocomial de la infección (OR=16.5 IC 95\% 5.6-49). Esta variable también fue estadísticamente significativa en los estudios más recientes de Cheol In kang (2010 y 2011) y Rodríguez-Baño (2010).

El estudio que más asociaciones encontró en el análisis multivariado fue el realizado por Rodríguez Baño en Sevilla (España) entre 2001 y 2005 sobre factores de riesgo asociados a bacteremia en 43 pacientes y el doble número de controles. Encontraron asociación con significancia estadística para las variables seguimiento como paciente ambulatorio, Índice de Charlson $>2$, daño estructural de la vía urinaria o biliar, catéter urinario y uso previo de oxy iminocefalosporinas, aminopenicilinas y fluoroquinolonas.

Para el uso de dispositivos médicos la mayor asociación se encontró en el estudio de Kuster para la variable ventilación mecánica (OR 10.56, IC 95\% 1.06-579.10, $\mathrm{p}=0.042$ ) y en el estudio de Rodríguez-Baño del 2008 para la variable catéter vesical (3.9 IC 95\% 1.1-13.7). A pesar que varios estudios encontraron asociaciones en el análisis univariado con el uso de catéter venoso central, ninguna alcanzó la significancia estadística en el multivariado.

La asociación con el tiempo de estancia solo se encontró en los estudios de Tumbarello y Chaiwarith y con la estancia en la unidad de cuidado intensivo solo en el estudio de Cheol In Kang. El origen nosocomial de la infección se asoció a la infección por bacterias productoras de BLEE en cuatro estudios (Rango de OR entre 1,86 y 16,5). Otras variables con asociaciones significativas, encontradas en estudios únicos fueron: traqueostomía, sonda orogástrica, edad, hogar geriátrico, transplante de órgano, anemia y enfermedad hepática de base. Ningún reporte encontró asociación con la diabetes, insuficiencia renal, enfermedad pulmonar crónica o inmunosupresión.Los estudios de
Cordery y Hyle tuvieron resultados negativos, es decir no encontraron ninguna asociación.

Las asociaciones encontradas en esta revisión difieren según el lugar donde fueron realizados los estudios. Las diferencias encontradas pueden ser originadas en las variables epidemiológicas locales, en el tamaño reducido de la muestra de algunos estudios, en la frecuencia en el uso de quinolonas en los países, en los criterios de apareamiento de los controles y en el sesgo de memoria que resta confiabilidad en la recolección de los antecedentes de los pacientes como el uso previo de antibióticos en el año anterior al momento en que se presentó la infección.

A pesar del número de publicaciones de los últimos años, se requieren de más estudios de factores de riesgo principalmente en Latinoamérica, con una muestra mayor que permita la construcción de una escala de riesgo que pueda ser aplicada en la evaluación inicial de los pacientes infectados con el fin de optimizar el uso empírico de antibióticos que disminuya el riesgo de mortalidad y limite el empleo de carbapenémicos. En nuestro conocimiento esta es la primera revisión sistemática publicada sobre factores de riesgo asociados con infección por BLEE.

\section{Referencias}

I. Villegas MV, Kattan JN, Quinteros MG, Casellas JM. Prevalence of extendedspectrum beta-lactamases in South America. Clin Microbiol Infect. 2008;14 Suppl 1:154-8.

2. Schwaber MJ, Navon-Venezia S, Kaye KS, Ben-Ami R, Schwartz D, Carmeli Y. Clinical and economic impact of bacteremia with extended- spectrum-betalactamase-producing Enterobacteriaceae. Antimicrob Agents Chemother. 2006;50: 1257-62.

3. Paterson DL, Bonomo RA. Extended-spectrum beta-lactamases: a clinical update. Clin Microbiol Rev. 2005; 18:657-86.

4. Bush K, Jacoby GA. Updated functional classification of beta-lactamases. Antimicrob Agents Chemother. 2010;54:969-76

5. Gunseren F, Mamikoglu L, Ozturk S, et al. A surveillance study of antimicrobial resistance of gram-negative bacteria isolated from intensive care units in eight hospitals in Turkey. J Antimicrob Chemother. 1999;43:373-8.

6. Hanberger H, Garcia-Rodriguez JA, Gobemado M, Goossens H, Nilsson LE, Struelens MJ. Antibiotic susceptibility among aerobic gram-negative bacilli in intensive care units in 5 European countries. French and Portuguese ICU Study Groups. JAMA. 1999;281:67-71

7. Albertini MT, Benoit C, Berardi L, et al. Surveillance of methicillin-resistant Staphylococcus aureus (MRSA) and Enterobacteriaceae producing extendedspectrum beta-lactamase (ESBLE) in Northerm France: a five-year multicentre incidence study. J Hosp Infect. 2002;52:107-13. 
8. Jacoby GA, Medeiros AA, O'Brien TF, Pinto ME, Jiang H. Broad-spectrum, transmissible beta-lactamases. N Engl J Med. 1988;319:723-4.

9. Moland ES, Black JA, Ourada J, Reisbig MD, Hanson ND, Thomson KS. Occurrence of newer beta-lactamases in Klebsiella pneumoniae isolates from 24 U.S. hospitals. Antimicrob Agents Chemother. 2002:46:3837-42.

10. National Nosocomial Infections Surveillance S. National Nosocomial Infections Surveillance (NNIS) System Report, data summary from January 1992 to June 2002, issued August 2002. Am J Infect Control. 2002;30:458-75

11. Bell JM. Turnidge JD, Gales AC, Pfaller MA, Jones RN, Sentry ASG. Prevalence of extended spectrum beta-lactamase (ESBL)-producing clinical isolates in the Asia-Pacific region and South Africa: regional results from SENTRY Antimicrobial Surveillance Program (1998-99). Diagn Microbiol Infect Dis. 2002;42:193-8.

12. Lewis MT, Yamaguchi $\mathrm{K}$, Biedenbach DJ, Jones RN. In vitro evaluation of ce fepime and other broad-spectrum beta-lactams in 22 medical centers in Japan: a phase II trial comparing two annual organism samples. The Japan Antimicrobial Resistance Study Group. Diagn Microbiol Infect Dis. 1999;35:307-15.

13. Yamaguchi K, Mathai D, Biedenbach DJ, Lewis MT, Gales AC, Jones RN Evaluation of the in vitro activity of six broad-spectrum beta-lactam antimicrobial agents tested against over 2,000 clinical isolates from 22 medical centers in Japan. Japan Antimicrobial Resistance Study Group. Diagn Microbiol Infect Dis. 1999;34:123-34

14. Winokur PL, Canton R, Casellas JM, Legakis N. Variations in the prevalence of strains expressing an extended-spectrum beta-lactamase phenotype and characterization of isolates from Europe, the Americas, and the Westem Pacific region. Clin Infect Dis. 2001;32 Suppl 2:S94-103.

15. Mendes C, Hsiung A, Kiffer C, et al. Evaluation of the in vitro activity of 9 antimicrobials against bacterial strains isolated from patients in intensive care units in brazil: MYSTIC Antimicrobial Surveillance Program. Braz J Infect Dis. 2000;4:236-44.

16. Miranda MC, Perez F, Zuluaga T, et al. [Antimicrobial resistance in gran negative bacteria isolated from intensive care units of Colombian hospitals, WHO NET 2003, 2004 and 2005]. Biomedica. 2006:26:424-33.

17. Otman J, Cavassin ED, Perugini ME, Vidotto MC. An outbreak of extendedspectrum beta-lactamase-producing Klebsiella species in a neonatal intensive care unit in Brazil. Infect Control Hosp Epidemiol. United States; 2002:8-9.

18. Pfaller MA, Jones RN, Doem GV. Multicenter evaluation of the antimicrobial activity for six broad-spectrum beta-lactams in Venezuela: comparison of data from 1997 and 1998 using the Etest method. Venezuelan Antimicrobial Resistance Study Group. Diagn Microbiol Infect Dis. 1999;35:153-8.

19. Sader HS, Jones RN, Gales AC, et al. Antimicrobial susceptibility patterns for pathogens isolated from patients in Latin American medical centers with a diag nosis of pneumonia: analysis of results from the SENTRY Antimicrobial Surveillance Program (1997). SENTRY Latin America Study Group. Diagn Microbiol Infect Dis. 1998;32:289-301.

20. Sader HS, Gales AC, Granacher TD, Pfaller MA, Jones RN. Group SS. Prevalence of antimicrobial resistance among respiratory tract isolates in Latin America: results from SENTRY antimicrobial surveillance program (1997-98). Braz J Infect Dis. 2000;4:245-54.

21. Villegas MV, Correa A, Perez F, et al. Prevalence and characterization of ex tended-spectrum beta-lactamases in Klebsiella pneumoniae and Escherichia coli isolates from Colombian hospitals. Diagn Microbiol Infect Dis. 2004;49:217-22.

22. Espinal P, Garza-Ramos U, Reyna F, et al. Identification of SHV-type and CTXM-12 extended-spectrum beta-lactamases (ESBLs) in multiresistant Enterobacteriaceae from Colombian Caribbean hospitals. J Chemother. 2010;22:160-4.

23. Tumbarello M, Spanu T, Sanguinetti M, et al. Bloodstream infections caused by extended-spectrum-beta-lactamase-producing Klebsiella pneumoniae: risk factors, molecular epidemiology, and clinical outcome. Antimicrob Agents Chemother. 2006;50:498-504

24. Superti SV, Augusti G, Zavascki AP. Risk factors for and mortality of extended-spectrum-beta-lactamase-producing Klebsiella pneumoniae and Escherichia coli nosocomial bloodstream infections. Rev Inst Med Trop Sao Paulo. 2009;51:211-6.
25. Cordery RJ, Roberts CH, Cooper SJ, Bellinghan G, Shetty N. Evaluation of risk factors for the acquisition of bloodstream infections with extended-spectrum beta-lactamase-producing Escherichia coli and Klebsiella species in the intensive care unit; antibiotic management and clinical outcome. J Hosp Infect. 2008:68:108-15.

26. Chenoweth CE, Saint S. Urinary tract infections. Infect Dis Clin North Am. 2011:25:103-15.

27. Saint S, Chenoweth CE. Biofilms and catheter-associated urinary tract infections Infect Dis Clin North Am. 2003;17:411-32.

28. Warren JW, Damron D, Tenney JH, Hoopes JM, Deforge B, Muncie HL, Jr Fever, bacteremia, and death as complications of bacteriuria in women with longterm urethral catheters. J Infect Dis. 1987;155:1 151-8.

29. Classen DC, Larsen RA, Burke JP, Stevens LE. Prevention of catheter-associated bacteriuria: clinical trial of methods to block three known pathways of infection. Am J Infect Control. 1991;19:136-42.

30. Garibaldi RA, Burke JP, Britt MR, Miller MA, Smith CB. Meatal colonization and catheter-associated bacteriuria. N Engl J Med. 1980;303:316-8.

31. Hartstein AI, Garber SB, Ward TT, Jones SR, Morthland VH. Nosocomial urinary tract infection: a prospective evaluation of 108 catheterized patients. Infect Control. 1981;2:380-6.

32. Kunin CM, McCormack RC. Prevention of catheter-induced urinary-tract infections by sterile closed drainage. N Engl J Med. 1966;274:1155-61.

33. Hooton TM, Bradley SF, Cardenas DD, et al. Diagnosis, prevention, and treatment of catheter-associated urinary tract infection in adults: 2009 Intemational Clinical Practice Guidelines from the Infectious Diseases Society of America. Clin Infect Dis. 2010;50:625-63.

34. Maki DG, Tambyah PA. Engineering out the risk for infection with urinary catheters. Emerg Infect Dis. $2001 ; 7: 342-7$.

35. Huth TS, Burke JP, Larsen RA, Classen DC, Stevens LE. Randomized trial of meatal care with silver sulfadiazine cream for the prevention of catheter-associated bacteriuria. J Infect Dis. 1992;165:14-8.

36. Platt R, Polk BF, Murdock B, Rosner B. Risk factors for nosocomial urinary tract infection. Am J Epidemiol. 1986;124:977-85.

37. Anderson DJ, Kirkland KB, Kaye KS, et al. Underresourced hospital infection control and prevention programs: penny wise, pound foolish? Infect Control Hosp Epidemiol. 2007;28:767-73.

38. Jarvis WR. Selected aspects of the socioeconomic impact of nosocomial infections: morbidity, mortality, cost, and prevention. Infect Control Hosp Epidemiol. 1996:17:552-7.

39. Stone PW, Braccia D, Larson E. Systematic review of economic analyses of health care-associated infections. Am J Infect Control. 2005;33:501-9.

40. Edwards JR, Peterson KD, Andrus ML, et al. National Healthcare Safety Network (NHSN) Report, data summary for 2006 through 2007, issued November 2008. Am J Infect Control. 2008;36:609-26.

41. Maki DG, Cobb L, Garman JK, Shapiro JM, Ringer M, Helgerson RB. An attachable silver-impregnated cuff for prevention of infection with central venous catheters: a prospective randomized multicenter trial. Am J Med. 1988:85:307-14.

42. Weber DJ, Rutala WA. Central line-associated bloodstream infections: prevention and management. Infect Dis Clin North Am. 2011;25:77-102.

43. Baker AM, Meredith JW, Haponik EF. Pneumonia in intubated trauma patients Microbiology and outcomes. Am J Respir Crit Care Med. 1996;153:343-9.

44. Craig CP, Connelly S. Effect of intensive care unit nosocomial pneumonia on duration of stay and mortality. Am J Infect Control. 1984;12:233-8.

45. Cunnion KM, Weber DJ, Broadhead WE, Hanson LC, Pieper CF, Rutala WA Risk factors for nosocomial pneumonia: comparing adult critical-care populations. Am J Respir Crit Care Med. 1996;153:158-62.

46. Jourdain B, Novara A, Joly-Guillou ML, et al. Role of quantitative cultures of endotracheal aspirates in the diagnosis of nosocomial pneumonia. Am J Respir Crit Care Med. 1995; 152:241-6. 
47. Warren DK, Shukla SJ, Olsen MA, et al. Outcome and attributable cost of ventilator-associated pneumonia among intensive care unit patients in a suburban medical center. Crit Care Med. 2003;31:1312-7.

48. Boyce JM, Potter-Bynoe G, Dziobek L, Solomon SL. Nosocomial pneumonia in Medicare patients. Hospital costs and reimbursement patterns under the prospective payment system. Arch Intern Med. 1991;151:1109-14.

49. van Nieuwenhoven CA, Buskens E, Bergmans DC, van Tiel FH, Ramsay G, Bonten MJ. Oral decontamination is cost-saving in the prevention of ventilatorassociated pneumonia in intensive care units. Crit Care Med. 2004;32:126-30.

50. Cook DJ, Walter SD, Cook RJ, et al. Incidence of and risk factors for ventilatorassociated pneumonia in critically ill patients. Ann Intern Med. 1998;129:43340 .

51. Stonecypher K. Ventilator-associated pneumonia: the importance of oral care in intubated adults. Crit Care Nurs Q. 2010;33:339-47.

52. Crnich CJ, Safdar N, Maki DG. The role of the intensive care unit environment in the pathogenesis and prevention of ventilator-associated pneumonia. Respir Care. 2005;50:813-36; discussion 36-8.

53. O'Keefe-McCarthy S. Evidence-based nursing strategies to prevent ventilatoracquired pneumonia. Dynamics. 2006;17:8-1 1 .

54. Safdar N, Crnich CJ, Maki DG. The pathogenesis of ventilator-associated pneumonia: its relevance to developing effective strategies for prevention. Respir Care. 2005;50:725-39; discussion 39-41.

55. Vincent JL, de Souza Barros D, Cianferoni S. Diagnosis, management and prevention of ventilator-associated pneumonia: an update. Drugs. 2010;70:1927-44.

56. Mendelson G, Hait V, Ben-Israel J, Gronich D, Granot E, Raz R. Prevalence and risk factors of extended-spectrum beta-lactamase-producing Escherichia coli and Klebsiella pneumoniae in an Israeli long-term care facility. Eur J Clin Microbiol Infect Dis. 2005;24:17-22

57. Wu UI, Yang CS, Chen WC, Chen YC, Chang SC. Risk factors for bloodstream infections due to extended-spectrum betalactamase-producing Escherichia coli. J Microbiol Immunol Infect. 2010;43:310-6.

58. Rodríguez-Baño J, Picon E, Gijon P, et al. Community-onset bacteremia due to extended-spectrum beta-lactamase-producing Escherichia coli: risk factors and prognosis. Clin Infect Dis. 2010;50:40-8.

59. Rodríguez-Baño J, Picon E, Gijon P, et al. Risk factors and prognosis of nosocomial bloodstream infections caused by extended-spectrum-beta-lactamaseproducing Escherichia coli. J Clin Microbiol. 2010;48:1726-31.

60. Rodríguez-Baño J, Navarro MD, Romero L, et al. Risk-factors for emerging bloodstream infections caused by extended-spectrum beta-lactamase-producing Escherichia coli. Clin Microbiol Infect. 2008;14:180-3.

61. Chaiwarith R, Pasogpakdee P, Salee P, Kanjanaratanakorn K, Sirisanthana T, Supparatpinyo K. Risk factors for extended-spectrum beta-lactamase-producing Klebsiella pneumoniae and Escherichia coli acquisition in a tertiary care teaching hospital in Thailand. J Hosp Infect. England. 2009:285-6.
62. Lin MF, Huang ML, Lai SH. Risk factors in the acquisition of extended-spectrum beta-lactamase Klebsiella pneumoniae: a case-control study in a district teaching hospital in Taiwan. J Hosp Infect. 2003;53:39-45.

63. Kuster SP, Hasse B. Huebner V, et al. Risks factors for infections with extendedspectrum beta-lactamase-producing Escherichia coli and Klebsiella pneumoniae at a tertiary care university hospital in Switzerland. Infection. 2010;38:33-40.

64. Kang CI, Song JH, Chung DR, et al. Risk factors and treatment outcomes of community-onset bacteraemia caused by extended-spectrum beta-lactamaseproducing Escherichia coli. Int J Antimicrob Agents. 2010;36:284-7.

65. Kang CI, Chung DR, Ko KS, Peck KR, Song JH, Korean Network for Study of Infectious D. Risk factors for infection and treatment outcome of extended-spectrum beta-lactamase-producing Escherichia coli and Klebsiella pneumoniae bacteremia in patients with hematologic malignancy. Ann Hematol. 2012;91:115-21.

66. Hyle EP, Lipworth AD, Zaoutis TE, et al. Risk factors for increasing multidrug resistance among extended-spectrumbeta-lactamase-producing Escherichia coli and Klebsiella species. Clin Infect Dis. 2005;40:1317-24.

67. Quirante OFn, Cerrato SG, Pardos SL. Risk factors for bloodstream infections caused by extended-spectrum 2-lactamase-producing Escherichia coli and Klebsiella pneumoniae. Braz J Infect Dis. 2011 Jul-Aug;15(4):370-6.

68. Ho PL, Chan WM, Tsang KW, Wong SS, Young K. Bacteremia caused by Escherichia coli producing extended-spectrum beta-lactamase: a case-control study of risk factors and outcomes. Scand J Infect Dis. 2002;34:567-73.

69. Peña C, Gudiol C, Tubau F, et al. Risk-factors for acquisition of extended-spectrum beta-lactamase-producing Escherichia coli among hospitalised patients. Clin Microbiol Infect. 2006; 12:279-84.

70. Garcia Hernandez A, Garcia-Vazquez E, Gomez Gomez J, Canteras M, Hernandez-Torres A, Ruiz Gomez J. [Predictive factors of ESBL versus non-ESBL Escherichia coli bacteraemia and influence of resistance on the mortality of the patients]. Med Clin (Barc). 2011;136:56-60.

71. Harris AD, McGregor JC, Johnson JA, et al. Risk factors for colonization with extended-spectrum beta-lactamase-producing bacteria and intensive care unit admission. Emerg Infect Dis. 2007;13:1144-9.

72. Du B, Long Y. Liu H, et al. Extended-spectrum beta-lactamase-producing Escherichia coli and Klebsiella pneumoniae bloodstream infection: risk factors and clinical outcome. Intensive Care Med. 2002;28:1718-23.

73. Wener KM, Schechner V, Gold HS, Wright SB, Carmeli Y. Treatment with fluoroquinolones or with beta-lactam-beta-lactamase inhibitor combinations is a risk factor for isolation of extended-spectrum-beta-lactamase-producing Klebsiella species in hospitalized patients. Antimicrob Agents Chemother. 2010;54:2010-6.

74. Velasco Arribas M, Barrena Puerta R, Asenjo Mota A. Valverde Canovas JF, Delgado-Iribarren A, Losa Garcia JE. [Risk factors for extended espectrum betalactamase producer E.coli bacteremia from urinary origin]. Med Clin (Barc). 2010;134:392-5. 